\title{
MicroRNA-212 facilitates the motility and invasiveness of esophageal squamous carcinoma cells
}

\author{
ZHI CHEN ${ }^{1,2^{*}}$, YUZHEN LIU ${ }^{1-3^{*}}$, BO QI ${ }^{1,2}$, CHENGWEI GU $^{1,2}$, XIUFENG WEI $^{1,2}$, \\ LING GUO ${ }^{1,2}$, WENJIAN YAO ${ }^{1,2}$ and BAOSHENG ZHAO ${ }^{1,2}$ \\ ${ }^{1}$ Department of Thoracic Surgery, ${ }^{2}$ Esophageal Cancer Institute, ${ }^{3}$ Henan Neurology Institute, \\ The First Affiliated Hospital of Xinxiang Medical University, Weihui, Henan 453100, P.R. China
}

Received July 30, 2018; Accepted August 5, 2019

DOI: $10.3892 / \mathrm{mmr} .2019 .10647$

\begin{abstract}
As a tumor-associated microRNA (miR), miR-212 has dual functions; either as an oncogene or a tumor suppressor. A high expression level of miR-212 was reported to be associated with poor outcome in patients with esophageal squamous cell carcinoma (ESCC), however, its role in ESCC progression has not been explored. In the present study, an in vitro cell model of lentivirus-mediated gain-of-function demonstrated promotion of ESCC cell migration and invasion when miR-212 was overexpressed, and no effect on cell proliferation. miR-212 resulted in downregulation of the expression of E-cadherin, $\beta$-catenin, vimentin and Twist1. Moreover, it led to increased levels of extracellular matrix (ECM)-degrading enzymes, matrix metalloproteinase-9 and urokinase-type plasminogen activator. Furthermore, berberine inhibited miR-212-induced ESCC cell migration, unlike the PI3K inhibitor LY294002, rapamycin (mTOR inhibitor), 5-(Tetradecyloxy)-2-furoic acid (TOFA; an acetyl-CoA carboxylase 1 inhibitor), metformin and propranolol. These data suggest that miR-212 activates multiple signaling cascades and facilitates ESCC cell motility and invasion by promoting the epithelial-mesenchymal transition and degrading the ECM. Berberine may be a potential therapeutic agent against metastasis in patients with ESCC, who express high levels of miR-212.
\end{abstract}

\section{Introduction}

Esophageal cancer (EC) is one of the most common types of cancer in the world, particularly in China (1). Two major

Correspondence to: Dr Baosheng Zhao, Department of Thoracic Surgery, The First Affiliated Hospital of Xinxiang Medical University, 88 Jiankang Road, Weihui, Henan 453100, P.R. China

E-mail: drbszhao@xxmu.edu.cn

*Contributed equally

Key words: microRNA-212, esophageal squamous cell carcinoma, migration, invasion, epithelial-mesenchymal transition, extracellular matrix-degrading enzyme subtypes of esophageal cancer are esophageal squamous cell carcinoma (ESCC), and adenocarcinoma. The International Agency for Research on Cancer reported that ESCC was most common in South-Eastern and Central Asia (79\% of the total global EC cases) and China had about half of all ESCC cases on worldwide in 2012 (2). Despite advances in diagnosis and treatments, ESCC continues to have poor prognosis due to progressive metastasis (3). The molecular mechanisms of ESCC invasion leading to metastasis remain poorly understood. Therefore, understanding the molecular mechanisms of progressive metastasis will help to ascertain promising therapeutic targets that may benefit the development of alternative and novel approaches in ESCC therapy.

MicroRNAs (miRNAs/miRs) constitute a class of endogenous small non-coding RNAs that regulate post-transcriptional suppression or translational repression of their target mRNAs $(4,5)$. Accumulating evidence indicates the essential roles of miRNAs in the initiation and progression of several types of cancer in humans (6,7). A number of miRNA expression profiling studies have demonstrated that miRNA expression signatures are associated with ESCC development and progression $(8,9)$. miRNA profiling of primary esophageal cancer tissue and paired paracancerous tissue of patients with ESCC demonstrated an association between four miRNAs and patient outcomes (10). One of these miRNAs was miR-212. High miR-212 expression was associated with short-term survival and poor clinical outcome in patients with ESCC (11), indicating that this miRNA may contribute to the development and metastasis of ESCC. However, the biological functions of miR-212 in ESCC progression remain unknown.

In the present study, the aim was to explore the roles of miR-212 in ESCC cell proliferation, migration and invasion, and the potential underlying mechanisms. Using the in vitro cell model of lentivirus-mediated gain-of-function, it was demonstrated that miR-212 had no significant effect on ESCC cell proliferation; rather, miR-212 facilitated migration and invasion of ESCC cells by promoting the epithelial-mesenchymal transition (EMT) process and increasing the protein levels of the extracellular matrix (ECM)-degrading enzymes matrix metalloproteinase (MMP)2, MMP9 and urokinase-type plasminogen activator (uPa).

The miR-212 level was previously associated with poor outcome in patients with ESCC (11), thus the potential of 
therapeutic drugs that inhibit the miR-212-induced ESCC cell migration was investigated. A number of drugs, including LY294002 (PI3K inhibitor) (12), rapamycin (mTOR inhibitor) (13), 5-(Tetradecyloxy)-2-furoic acid (TOFA; an acetyl-CoA carboxylase 1 inhibitor) (14), metformin (15), propranolol (16) and berberine (17), were chosen based on: i) The classical signaling molecules that are associated with cancer development, such as the PI3K-Akt/mTOR-p70S6K and AMP-activated protein kinase (AMPK) pathways (12-14); ii) drugs, such as metformin and propranolol, that have demonstrated anti-tumor functions in several types of cancer; and iii) drugs, such as berberine, that demonstrated an inhibitory function against ESCC cell proliferation and migration (17). It was found that only berberine, an alkaloid extracted from Ranunculaceae coptis rhizome, and none of the other tested drugs, displayed an inhibitory function against miR-212 overexpression-induced ESCC cell migration.

\section{Materials and methods}

Reagents and antibodies. All-in-One $\mathrm{e}^{\mathrm{TM}}$ miRNA first-strand cDNA synthesis kit, All-in-One ${ }^{\mathrm{TM}}$ miRNA qPCR detection kit and all quantitative (q)PCR primers were purchased from GeneCopoeia, Inc. Primerscript ${ }^{\mathrm{TM}}$ RT reagent kit with gDNA eraser and SYBR premix Ex Taq II were obtained from Takara Biotechnology Co., Ltd. 3-(4,5-Dimethylthiazol-2-yl)-2,5diphenyltetrazolium bromide (MTT) was purchased from Beyotime Institute of Biotechnology. The cell culture media, including RPMI-1640 medium and DMEM, were obtained from Corning Inc. Fetal bovine serum (FBS) was purchased from Clark Bioscience. All primary antibodies, including those against E-cadherin (3195), $\beta$-catenin (8480), vimentin (5741), Twist1 (46702) and GAPDH (5174) were purchased from Cell Signaling Technology, Inc. The secondary antibody used was horseradish peroxidase-labeled goat anti-rabbit IgG $(\mathrm{H}+\mathrm{L}$; A0208) obtained from Beyotime Institute of Biotechnology. Berberine hydrochloride was purchased from Shanghai YuanYe Biotechnology Co., Ltd. Metformin and rapamycin were purchased from Selleck Chemicals. Propranolol was obtained from Sigma-Aldrich (Merck KGaA), LY294002 was ordered from Beyotime Institute of Biotechnology, and TOFA was purchased from Santa Cruz Biotechnology, Inc.

Cell culture and lentivirus infection. Three human ESCC cell lines KYSE-450, TE-1 and Eca109 were obtained from Cobioer Biosciences, Co., Ltd. KYSE-450 and TE-1 cells were cultured in RPMI-1640 medium, containing 10\% FBS, and Eca109 was cultured in DMEM supplemented with $10 \% \mathrm{FBS}$ at $37^{\circ} \mathrm{C}$ in $5 \% \mathrm{CO}_{2}$. All culture media contained $100 \mu \mathrm{g} / \mathrm{ml}$ penicillin and $100 \mu \mathrm{g} / \mathrm{ml}$ streptomycin (Beijing Solarbio Science \& Technology Co., Ltd.).

Viral particles of lentiviral vectors LV10-(U6/RFP \& Puro) expressing a scrambled control (Con, 5'-TTCTCCGAACGT GTCACGT-3') and mature hsa-miR-212 (miR-212, 5'-ACC UUGGCUCUAGACUGCUUACU-3') were purchased from Shanghai GenePharma Co., Ltd. The viral titers of miR-212and the Con-expressing vectors were at a concentration of $1 \times 10^{8}$ transduction $(\mathrm{T}) \mathrm{U} / \mathrm{ml}$. Cells $\left(2 \times 10^{5}\right)$ were seeded into a $3.5-\mathrm{cm}$ dish for viral infection. The cells were infected with $0,10,20$ and $40 \mu 1$ lentivirus to determine the multiplicity of infection (MOI). The MOI was 0, 5, 10 and 20 when the cells were infected with $0,10,20$ and $40 \mu l$ lentivirus, respectively. Based on the cell proliferation and migration for each MOI, $20 \mu \mathrm{l}$ lentivirus or MOI 10 was chosen throughout the present study. Cells were cultured in 6-well plate in medium with $5 \%$ FBS for approximately $24 \mathrm{~h}$, and subsequently infected with $20 \mu \mathrm{l}$ Con and miR-212 vector-expressing viral particles, in the presence of $5 \mu \mathrm{g} / \mathrm{ml}$ polybrene. At $6 \mathrm{~h}$ after infection, the supernatant was removed. After $24 \mathrm{~h}$ transfection, puromycin ( $5 \mu \mathrm{g} / \mathrm{ml}$ ) was added to the culture medium every 3 days for a selection of successful transfected cells to become a population of stable cells expressing miR-212 or scrambled control. Unless otherwise specified, all the cells used in the subsequent experiments were those stably expressing miR-212 after lentivirus transfection.

Cell proliferation assay. An MTT assay was performed to measure miR-212-overexpressed cell proliferation. Briefly,

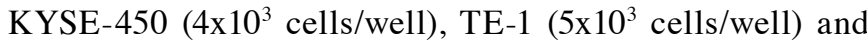

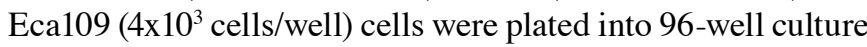
plates and incubated overnight at $37^{\circ} \mathrm{C}$ in $5 \% \mathrm{CO}_{2}$. MTT $(10 \mu \mathrm{l})$ was added to each well at a final concentration of $5 \mathrm{mg} / \mathrm{ml}$ at $0,24,48$, and $72 \mathrm{~h}$. After $4 \mathrm{~h}$ incubation at $37^{\circ} \mathrm{C}$, the blue MTT formazan crystals were dissolved in $100 \mu \mathrm{l} /$ well DMSO. The absorbance at $490 \mathrm{~nm}$ was measured via the Multiskan spectrum microplate reader (Thermo Fisher Scientific, Inc.). All experiments were repeated three times.

Cell migration and invasion assays. Cell migration and invasion were assayed using a 6.5-mm diameter Transwell chamber with $8-\mu \mathrm{m}$ micropores (Corning Inc.). For invasion assays, the upper chamber was pre-coated with $50 \mu$ l Matrigel $(1 \mathrm{mg} / \mathrm{ml})$ at $37^{\circ} \mathrm{C}$ for $8 \mathrm{~h}$. Then, the Matrigel was removed from the chamber. For migration and invasion assays, $200 \mu \mathrm{l}$ of the cell suspension ( $1 \times 10^{5}$ cells $/ \mathrm{ml}$ in serum-free medium) was placed in the upper chamber without serum, and $600 \mu \mathrm{l}$ of $10 \%$ FBS-containing medium was added to the bottom chamber. For the drug treatment experiments, the drug at final concentration indicated below was added to the cell suspension. After incubation for $36 \mathrm{~h}$, cells were fixed with $4 \%$ paraformaldehyde for $15 \mathrm{~min}$ at room temperature and then stained with $0.1 \%$ crystal violet for another $15 \mathrm{~min}$. Non-migrated or non-invaded cells on the upper surface of the chambers were cleaned with a cotton swab, and migrated cells or invaded cells in five different fields per chamber were counted using a phase contrast microscope under x100 magnification (Nikon TS100; Nikon Corporation).

Western blot analysis. Cells were washed with cold PBS and lysed with RIPA lysis buffer (Wuhan Boster Biological Technology, Ltd.). The protein concentration was determined by the BCA method. Protein $(30 \mu \mathrm{g})$ was subjected to SDS-PAGE on a $10 \%$ gel and transferred onto a nitrocellulose membrane (GE Healthcare). Following blocking with 5\% non-fat milk for $1 \mathrm{~h}$ at room temperature, the membrane was incubated at $4^{\circ} \mathrm{C}$ overnight with primary antibodies against each of the following proteins: E-cadherin, $\beta$-catenin, vimentin, Twist1 and GAPDH (all 1:1,000 dilution). Following washing, the membrane was incubated with corresponding horseradish peroxidase-conjugated secondary antibody (1:1,000 dilution) 
Table I. Sequences of the primers used in the reverse transcription-quantitative PCR experiments.

Gene (Acc. no.)

Primer sequence

MMP2 (NM_004530.5)

F: 5'-TTGACGGTAAGGACGGACTC-3'

MMP9 (NM_004994.2)

R: 5'-TCTCAAAGTTGTAGGTGGTGGA-3'

F: 5'-GACAGCGACAAGAAGTGGGG-3'

R: 5'-TCAGGGCGAGGACCATAGAG-3'

uPA (NM_002658.4)

F: 5'-CACACTGCTTCATTGATTACCCA-3'

R: 5'-AAGGCAATGTCGTTGTGGTG-3'

GAPDH (NM_002046)

F: 5'-GCAGGGGGGAGCCAAAAGGGT-3'

R: 5'-TGGGTGGCAGTGATGGCATGG-3'

MMP, matrix metalloproteinase; uPA, urokinase-type plasminogen activator; miR, microRNA.

for $1 \mathrm{~h}$ at room temperature. The membrane was washed and immersed in SuperSignal West Pico Chemiluminescent Substrate detection kit (Thermo Fisher Scientific, Inc.). Bound secondary antibody was detected by the Amersham ${ }^{\mathrm{TM}}$ Imager 600 system (GE Healthcare Bio-Sciences). Image J software v1.8.0.112 (National Institutes of Health) was used to quantify all protein band densities. The level of the tested proteins is represented by determining the ratio of the integrated density of tested protein relative to that of the GAPDH loading control.

RNA extraction and reverse transcription $(R T)-q P C R$. Total RNA was extracted using TRIzol reagent (Invitrogen; Thermo Fisher Scientific, Inc.). To detect miR-212 expression, cDNA was synthesized from $1 \mu \mathrm{g}$ total RNA by using All-in-One ${ }^{\mathrm{TM}}$ miRNA first-strand cDNA synthesis kit (GeneCopoeia, Inc.) according to the manufacturer's instructions. All-in-One ${ }^{\mathrm{TM}}$ miRNA qPCR kit (GeneCopoeia, Inc.) was used to amplify miR-212. The qPCR reaction was carried out for 40 cycles with the following temperature profile: Initial denaturation at $95^{\circ} \mathrm{C}$ for $10 \mathrm{~min}$, annealing at $62.5^{\circ} \mathrm{C}$ for $20 \mathrm{sec}$ and extension at $72^{\circ} \mathrm{C}$ for $10 \mathrm{sec}$. U6 was used as the internal control housekeeping gene. The primers of has-miR-212 and U6 were purchased from GeneCopoeia, Inc.

To detect the mRNA levels of MMP2, MMP9 and uPA, cDNA was synthesized from $1 \mu \mathrm{g}$ total RNA using PrimerScript $^{\mathrm{TM}}$ RT reagent kit with gDNA Eraser (Takara Biotechnology Co., Ltd.) following the manufacturer's instructions. The resulting cDNA was analyzed by RT-qPCR using the SYBR Premix Ex Taq II (Takara Biotechnology Co., Ltd.). The qPCR reaction was run by 40 cycles of a two-step thermocycling program: Denaturation at $95^{\circ} \mathrm{C}$ for $30 \mathrm{sec}$ and extension at $95^{\circ} \mathrm{C}$ for $5 \mathrm{sec}$. GAPDH was used as the internal control. The primer sequences for qPCRs are listed in Table I.

All qPCR reactions were run on a QuantStudio ${ }^{\mathrm{TM}}$ Dx Real-Time PCR Instrument (Applied Biosystems; Thermo Fisher Scientific, Inc.). The relative levels of miR-212 and indicated genes were normalized to the corresponding internal control and quantified based on the $2^{-\Delta \Delta \mathrm{Cq}}$ method (18).

Statistical analysis. All data are expressed as the mean \pm standard deviation and were analyzed using ANOVA. Levene's test was used to assess the equality of variances of each group. Tukey's test was used to analyze the equal variances while Games-Howell's test was used to assess the unequal variances in multiple comparisons. $\mathrm{P}<0.05$ was considered to indicate a statistically significant difference. All analyses were performed with SPSS version 22.0 (IBM Corp).

\section{Results}

miR-212 has no effect on ESCC cell proliferation. miR-212-overexpressing ESCC cell lines were generated by lentiviral infection to explore the function and the possible mechanism of miR-212 in ESCC cells. As indicated in Fig. 1, overexpression of miR-212 was confirmed by RT-qPCR. Therefore, this in vitro cell model was used to study the role of miR-212 in subsequent experiments.

The effect of overexpressing miR-212 on the proliferation of ESCC cells was investigated by MTT assay. As shown in Fig. 2, the cell proliferation of all three miR-212-overexpressing ESCC cell lines was similar to that of the corresponding control cell lines at all tested time points. These results indicate that miR-212 has no significant effect on ESCC cell proliferation.

miR-212 promotes ESCC cell migration and invasion. As high expression of miR-212 was associated with poor outcome in patients with ESCC (11), the effect of miR-212 on ESCC cell migration and invasion was examined using Transwell assays. As shown in Fig. 3, overexpression of miR-212 significantly increased cell migration (Fig. 3A and B) as well as invasion (Fig. 3C and D), compared with the corresponding controls. These results suggest that miR-212 plays a facilitative role in ESCC cell migration and invasion.

miR-212 overexpression promotes EMT and enhances the level of ECM-degrading enzymes in ESCC cells. Cancer cells often undergo EMT before gaining their metastatic potential (19). To elucidate further mechanistic insights into the role of miR-212 in promoting ESCC cell migration and invasion, western blot analyses were performed to examine the expression of EMT-associated markers. As shown in Fig. 4A and C, overexpression of miR-212 led to the decrease of E-cadherin (epithelial marker) and the increase of $\beta$-catenin, vimentin, and transcription factor Twist1 (mesenchymal markers). This indicates that miR-212 may promote EMT in ESCC cells. 

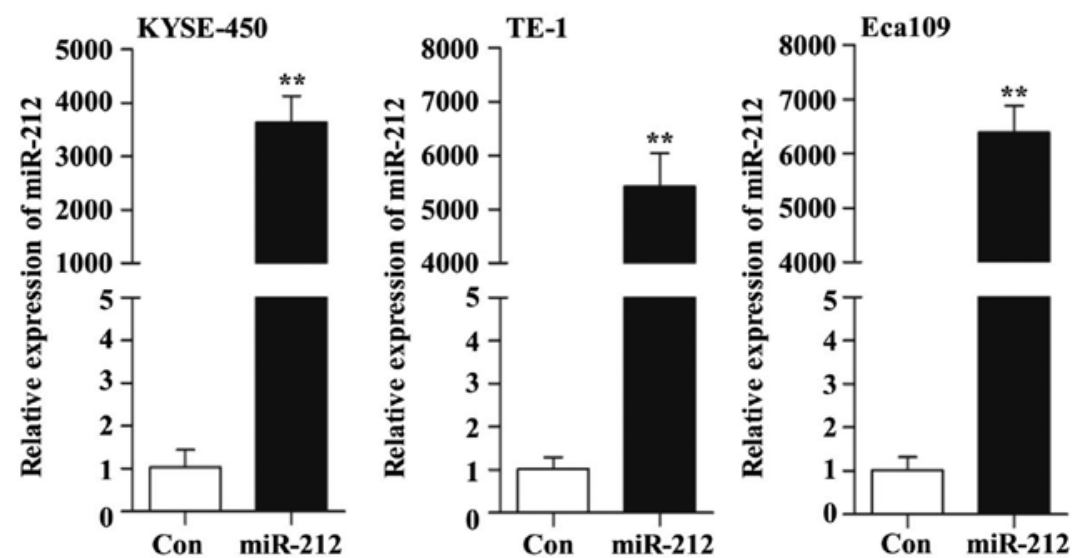

Figure 1. miR-212 is overexpressed in lentivirus-infected ESCC cells. The relative expression level of miR-212, after infection with either lentivirus-mediated miR-212 mimic (miR-212) or lentivirus-mediated Con into KYSE-450, TE-1 and Eca109 cells, was examined by reverse transcription-quantitative PCR. The y-axis represents the relative expression level of miR-212 in different virus-infected ESCC cell lines normalized to the levels in the Con cells. Data are presented as the mean \pm SD of three independent experiments. ${ }^{* *} \mathrm{P}<0.01$. ESCC, esophageal squamous cell carcinoma; miR, microRNA; Con, scrambled control.
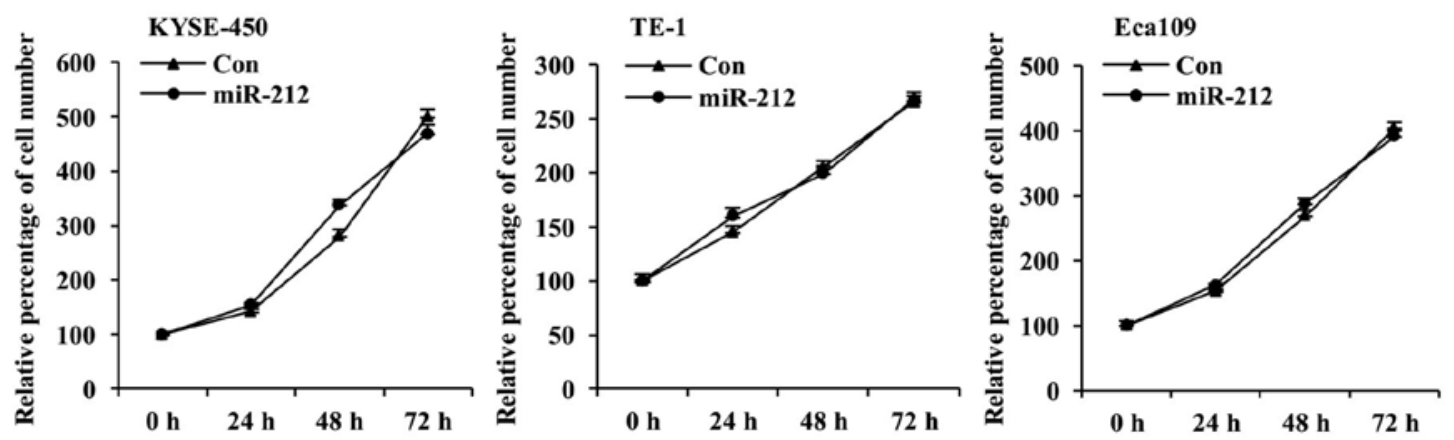

Figure 2. miR-212 overexpression does not affect ESCC cell proliferation. The proliferation of three miR-212-overexpressed ESCC KYSE-450, TE-1 and Eca109 cell lines was measured by MTT at 24, 48 and $72 \mathrm{~h}$. The y-axis is the percentage of the cell number at each time point relative to the $0 \mathrm{~h}$ time point in the respective group. Data are presented as the mean \pm SD of three independent experiments. ESCC, esophageal squamous cell carcinoma; miR, microRNA; Con, scrambled control.

Increased proteinase activity in the ECM surrounding malignant tumor cells has been implicated in metastatic processes (20). Hence, the protein expression of uPA, MMP2 and MMP9, which are key ECM proteinases and markers of cancer cell invasion (21), was examined. As shown in Fig. 4B, D and E, overexpression of miR-212 significantly increased the protein and mRNA levels of MMP9 and UPA in the three ESCC cell lines, compared with the corresponding controls. Interestingly, miR-212 did not cause any significant alteration of MMP2 protein and mRNA levels. Collectively, these findings indicate that miR-212-induced ESCC cell migration and invasion may be mediated by promoting EMT and increasing the level of ECM proteinases.

Berberine inhibits miR-212-induced migration of ESCC cells. As miR-212 was demonstrated to promote ESCC cell migration and invasion, the potential of therapeutic drugs against metastasis of ESCC with high miR-212 expression was explored. The effect of LY294002 (PI3K inhibitor), rapamycin (mTOR inhibitor), TOFA (acetyl-CoA carboxylase 1 inhibitor inhibitor), metformin, propranolol and berberine were tested. A Transwell migration assay was performed to detect the effect of these drugs on miR-212-induced ESCC cell migration. The concentrations of these drugs here used were based on preliminary results obtained from miR-212-overexpressed KYSE-450 cells and vector control KYSE-450 cells (data not shown). Compared with the vehicle-treated control cells, with the exception of berberine, none of these drugs caused changes in miR-212-induced KYSE-450 cell migration (Fig. 5), indicating that high motility of ESCC cells initiated by miR-212 may be triggered by activation of multiple signaling cascades.

It was previously shown that berberine, a traditional Chinese medicine, displayed antitumor activity against ESCC cells by regulating a number of signaling pathways (17). Accumulating evidence indicates that berberine prevents cell motility by inhibition of EMT in a number of cancer cell types including esophageal cancer cells $(22,23)$. Based on the results shown in Fig. 5, the inhibitory function of berberine on miR-212-induced cell migration was further investigated by focusing on miR-212-overexpressed KYSE-450 cells. The Transwell migration was conducted with or without berberine treatment. As shown in Fig. 6A, the intact images of the migrated cell on the lower surface of the chambers revealed that berberine had an inhibitory effect on miR-212-induced 
$\mathbf{A}$

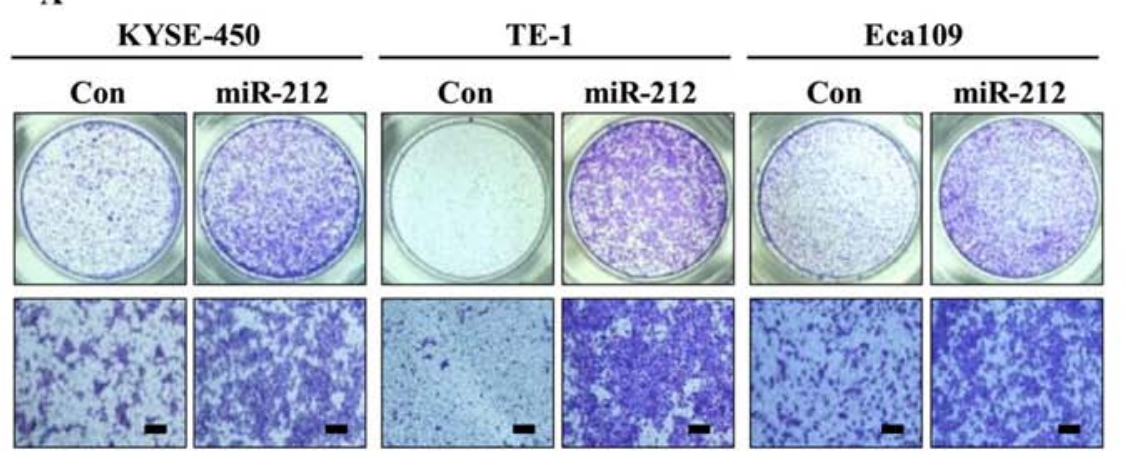

C
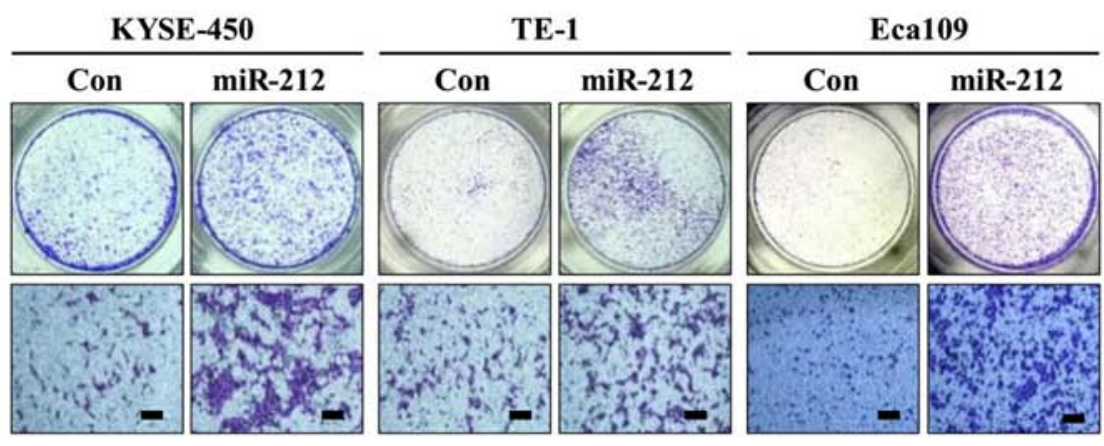

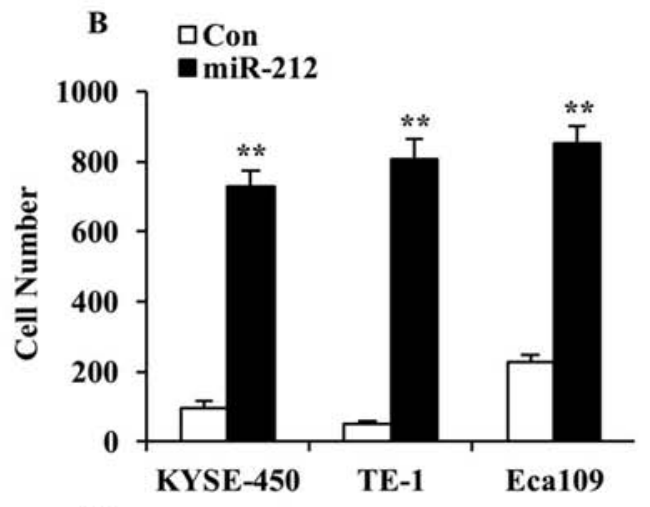

D

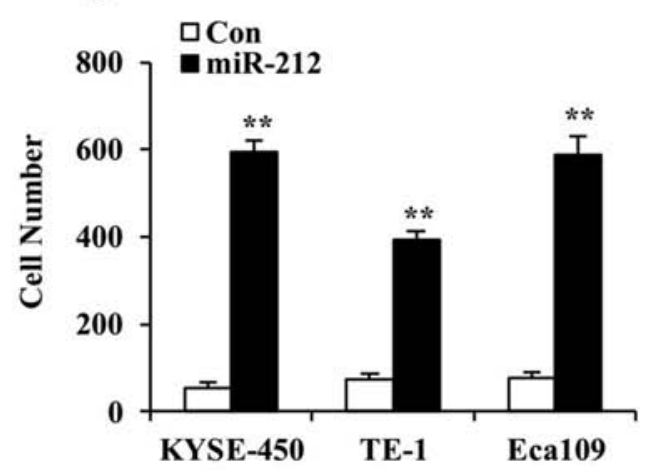

Figure 3. miR-212 overexpression promotes ESCC cell migration and invasion. (A) Representative images and (B) quantification of migrated cells measured in Transwell assays, using miR-212-overexpressed and Con-transfected ESCC KYSE-450, TE-1 and Eca109 cell lines. (C) Representative images and (D) quantification of Transwell invasion assays. Scale bars, $100 \mu \mathrm{m}$. Data are expressed as the mean $\pm \mathrm{SD} .{ }^{* *} \mathrm{P}<0.01$. ESCC, esophageal squamous cell carcinoma; miR, microRNA; Con, scrambled control.

cell migration when compared with that of DMSO treatment in miR-212-overexpressed KYSE-450 cells. Such inhibitory effect on cell migration was more evident with berberine at $15 \mu \mathrm{M}$ than $5 \mu \mathrm{M}$. In an effort to study whether blocking EMT is a potential mechanism by which berberine plays the inhibitory effect on miR-212-induced cell migration, western blotting was conducted to examine the protein levels of EMT-related signal markers. As shown in Fig. 6B and C, berberine treatment clearly augmented the level of E-cadherin and attenuated the levels of $\beta$-catenin and Twist1 when comparing with DMSO treatment in miR-212-overexpressed KYSE-450 cells. Taken together, these results imply that berberine prevents miR-212-induced ESCC cell migration by inhibiting EMT process.

\section{Discussion}

Accumulating evidence has indicated that miRNAs participate in the progression of several types of cancer by targeting multiple genes involved in cancer progression and metastasis, and the aberrant expression of miRNAs contributes to the pathogenesis of malignancies in humans (24). Thus, exploration of cancer-specific miRNAs could help identify novel biomarkers and therapeutic targets in cancer.

Human miR-212 (hsa-miR-212) shares close sequence homology with hsa-miR-132; both are encoded from the same intron of a small non-coding gene located on chromosome 17 in humans (25). The miR-132/miR-212 cluster was originally identified in neuronal cells and displayed important functions in neuronal development $(26,27)$. Although the roles of miR-132/212 have been investigated mainly in neurons, increasing evidence point to their involvement in cancer.

Despite previous observation of significant association between miR-212 and short-term survival and poor outcome in patients with ESCC due to early metastasis (12), the function and underlying mechanism of miR-212 remain unknown. In the present study, it was demonstrated that miR-212 had no effect on ESCC cell proliferation, but significantly promoted cell migration and invasion, possibly through facilitating EMT and increasing the level of ECM-degrading enzymes. Moreover, berberine may be used as an alternative therapy to effectively inhibit invasion and metastasis in patients with ESCC, who express high levels of miR-212.

Tumor cell metastasis is a complex multi-step process. An important event of metastasis is EMT, by which epithelial cells acquire mesenchymal phenotype, thereby obtaining the ability to migrate and invade. EMT is characterized by loss of epithelial markers and gain of mesenchymal markers. Several groups of transcription factors, including Snail and Twist families, repress the transcription of epithelial markers, such as E-cadherin, and promote the transcription of mesenchymal markers, such as $\beta$-catenin and vimentin. In the present study, overexpression of miR-212 attenuated E-cadherin expression, but augmented $\beta$-catenin, vimentin, and Twist 1 . This observation indicates that miR-212 promotes ESCC migration by activation of the EMT process. 

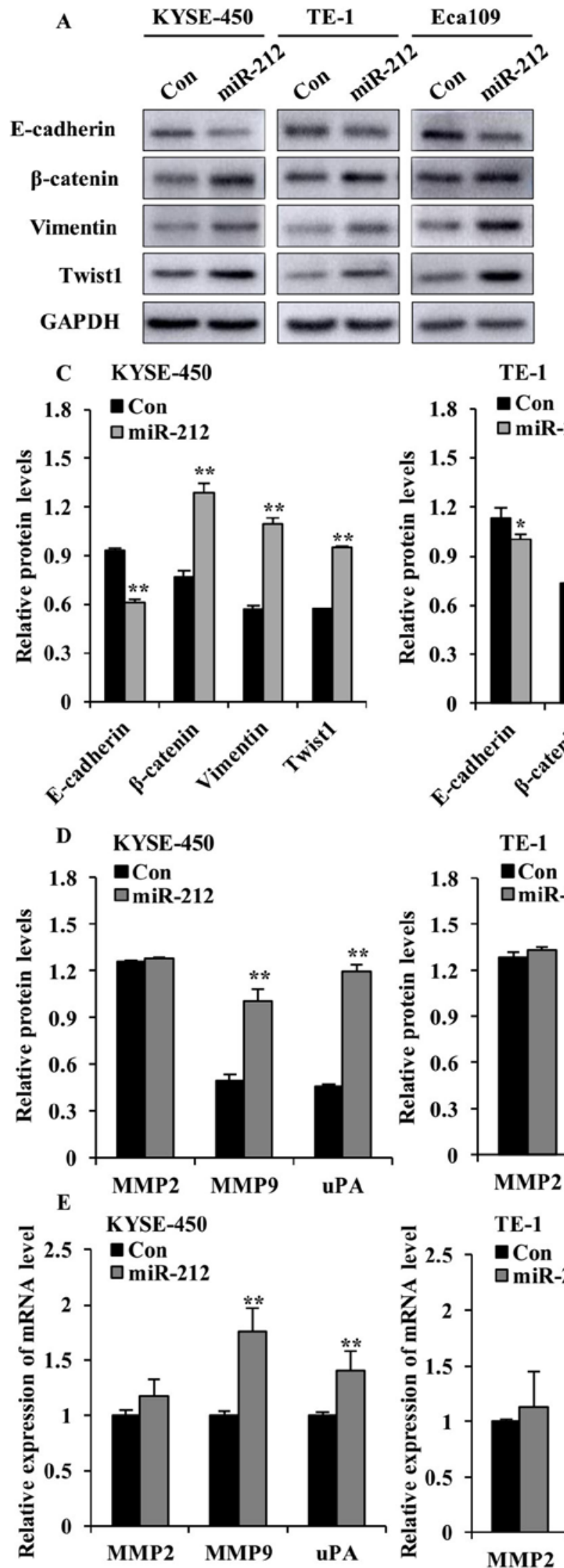

TE-1
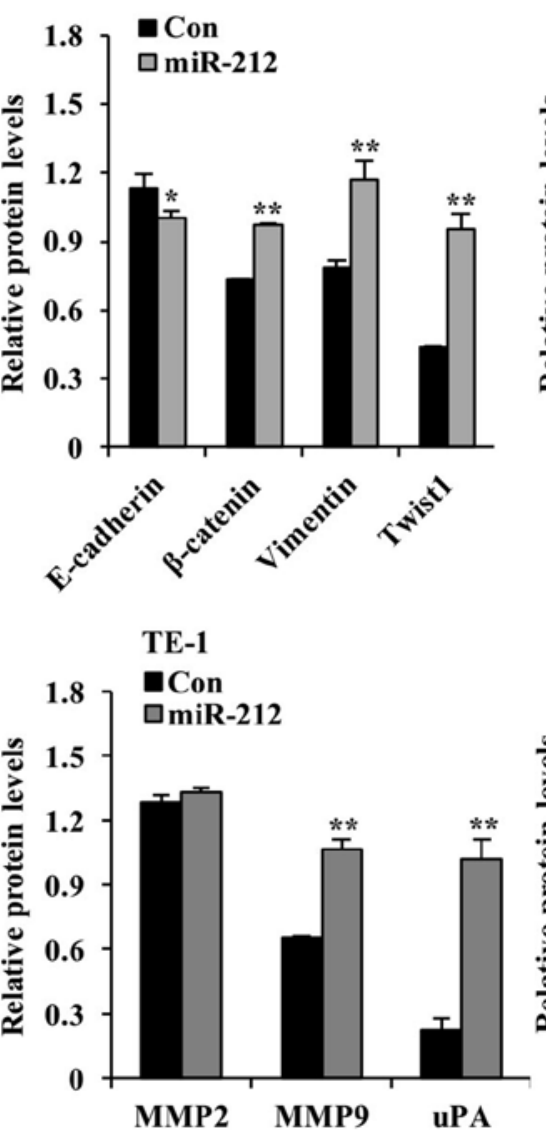

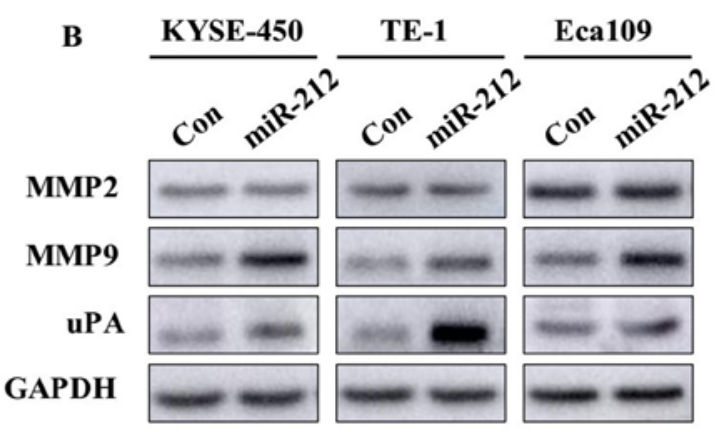

Eca109
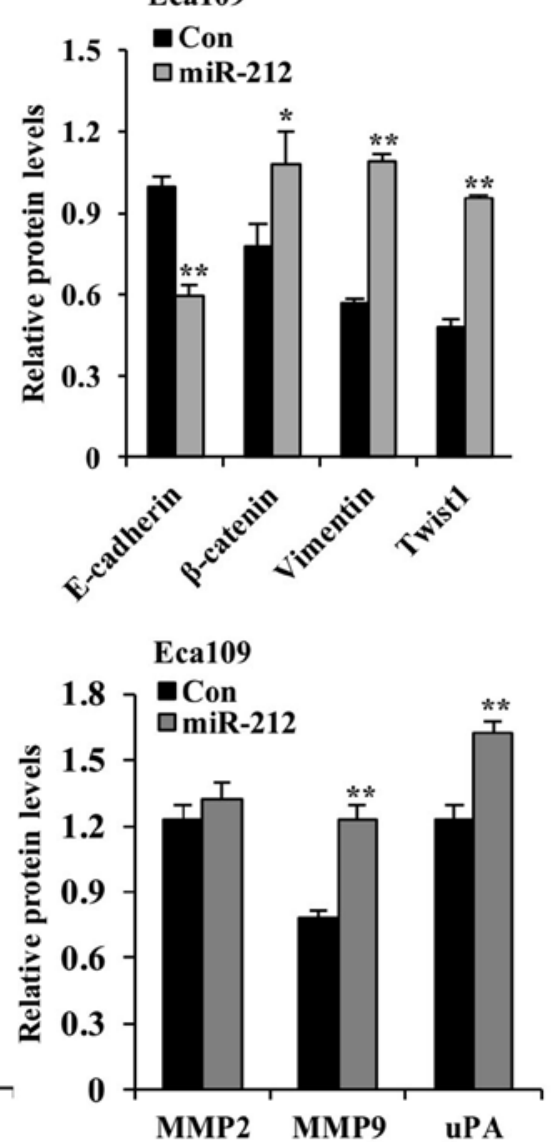
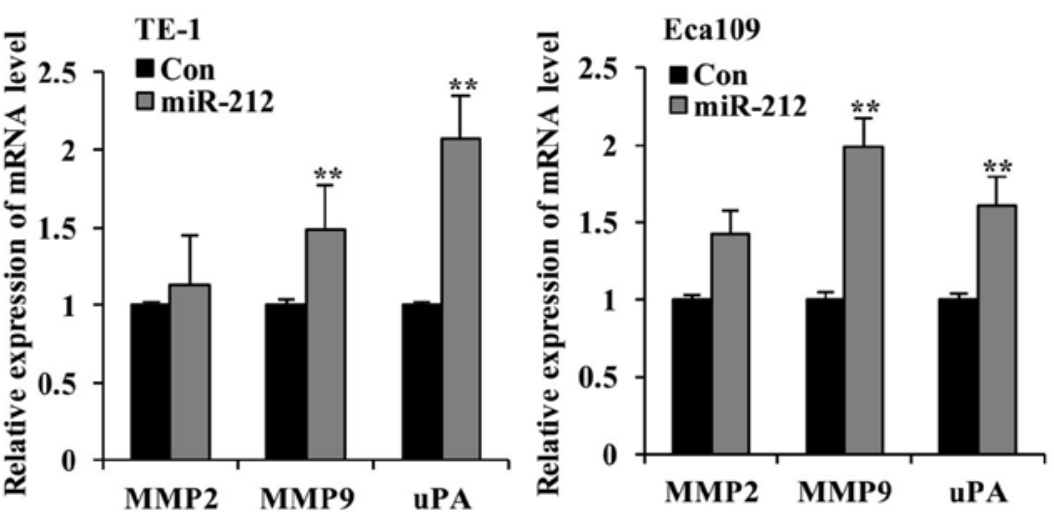

Figure 4. miR-212 overexpression enhances the levels of EMT markers, MMP2, MMP9 and uPA in ESCC cells. (A) Western blot analysis of the expression of E-cadherin, $\beta$-catenin, vimentin and Twist1, and (B) MMP2, MMP9 and uPA in miR-212-overexpressed (miR-212) and Con-transfected ESCC cells. (C) Quantification plots of the protein levels detected in (A). (D) Quantification plots of the protein levels detected in (B). The y-axis represents the ratio of the integrated density of tested protein relative to GAPDH loading control. (E) Reverse transcription-quantitative PCR results of mRNA levels of MMP2, MMP9 and uPA in miR-212-overexpressed and Con ESCC cells. The y-axis represents the relative mRNA level normalized to the level in the respective Con group. The relative mRNA level is the ratio of indicated mRNA level to the GAPDH level in each group. Data are expressed as mean \pm SD. ${ }^{*}<<0.05$ and ${ }^{* *} \mathrm{P}<0.01$ vs. the respective Con cells. ESCC, esophageal squamous cell carcinoma; miR, microRNA; Con, scrambled control; MMP, matrix metalloproteinase; uPA, urokinase-type plasminogen activator. 
A

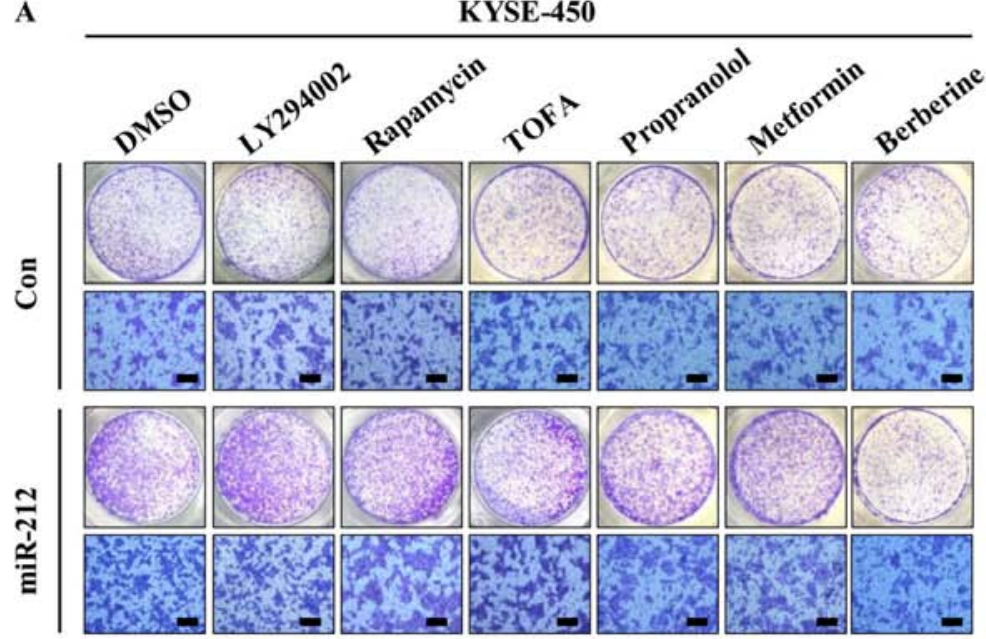

B

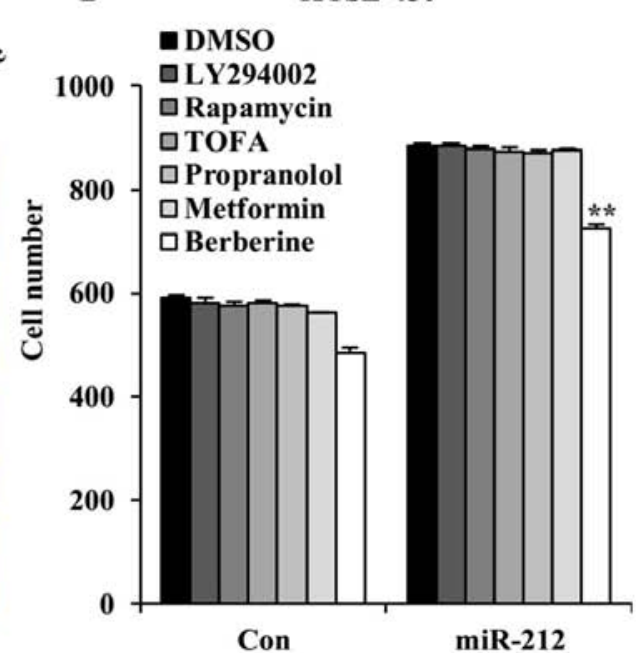

Figure 5. Effects of drugs on miR-212-induced migration in KYSE-450 cells. (A) Representative images of miR-212-overexpressed KYSE-450 cells and Con-transfected cells treated with metformin $(10 \mathrm{mM})$, rapamycin $(100 \mathrm{nM})$, propranolol $(50 \mu \mathrm{M}), \mathrm{LY} 294002(4 \mu \mathrm{M})$, TOFA $(1 \mu \mathrm{g} / \mathrm{ml})$ or berberine $(15 \mu \mathrm{M})$. (B) Quantification of migrated cells from Transwell assay. The data are presented as the mean $\pm \mathrm{SD}$. ${ }^{* \mathrm{P}} \mathrm{P}<0.01$ vs. the respective Con group. miR, microRNA; Con, scrambled control; TOFA, 5-(Tetradecyloxy)-2-furoic acid.

$\mathbf{A}$

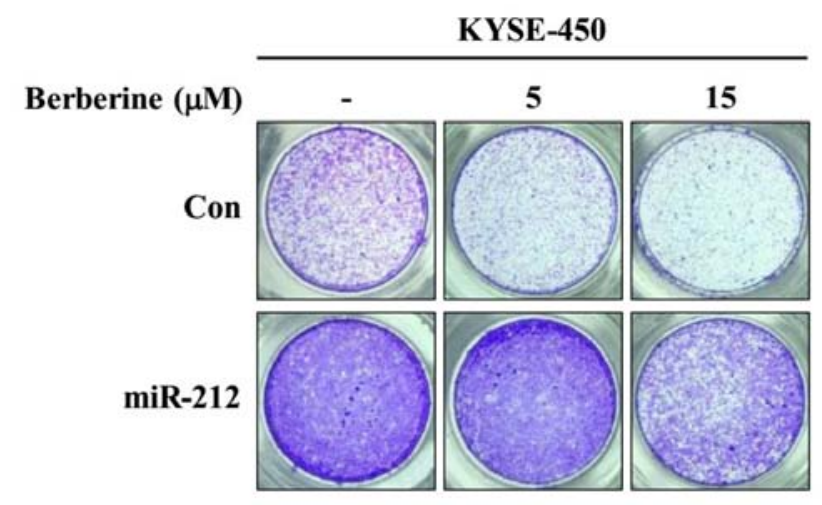

B

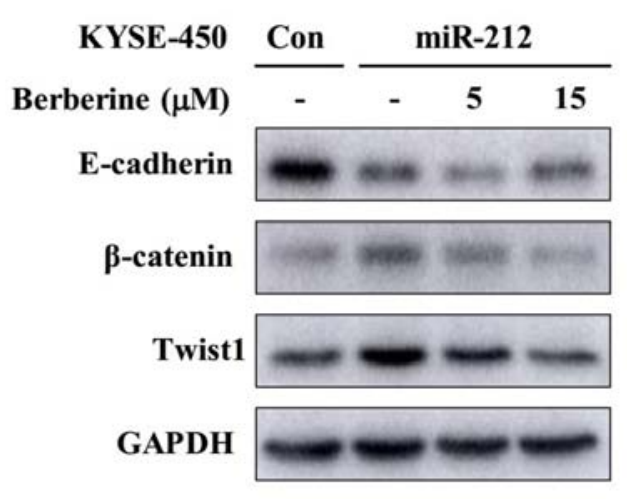

C KYSE-450

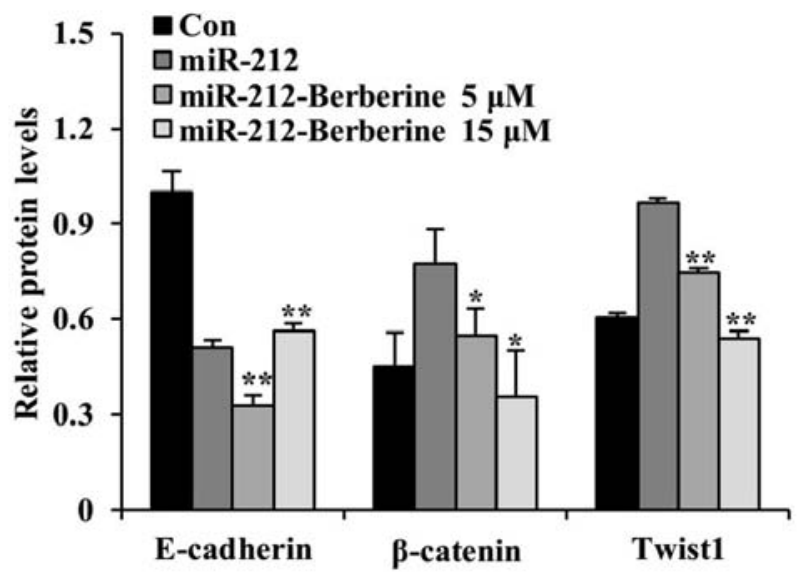

Figure 6. Berberine attenuates miR-212-induced KYSE-450 cell migration and EMT progression. (A) Representative images of migrated cells after treatment with 5 and $15 \mu \mathrm{M}$ berberine in a Transwell migration assay. (B) Western blot analysis of E-cadherin, $\beta$-catenin and Twist1 after cells were treated with berberine at for $24 \mathrm{~h}$. (C) Quantification of the relative EMT-associated protein levels, normalized to GAPDH. The data are presented as the mean \pm SD. ${ }^{*} \mathrm{P}<0.05$ and ${ }^{* *} \mathrm{P}<0.01$ vs. DMSO-treated miR-212 (miR-212). miR, microRNA; Con, scrambled control; EMT, epithelial-mesenchymal transition.

Degradation of ECM and basement membrane is critical for tumor invasion and metastasis. MMP2 and MMP9 are functionally related gelatinase/collagenase IV proteins and their expression and activity have been linked to metastasis or invasion in a majority of cancer types $(28,29)$. This led to the investigation of the effect of miR-212 on the expression 
of MMP2, MMP9 and UPA in ESCC cells. It was found that miR-212 significantly increased the protein and mRNA levels of MMP9 and UPA in three ESCC cell lines, with no alteration of MMP2 mRNA and protein levels. These findings suggest that miR-212 increases the expression of ECM-degrading enzymes to degrade the ECM, which allows ESCC cells to escape from the primary tumor and invade adjacent tissues, and to migrate to distant regions. It has been demonstrated that gene expression of MMP2 and MMP9 is regulated mainly at the transcription level and both are co-regulated to some extent in their expression due to their promoters sharing common structural features (30). However, distinct compositions have been identified between promoters of MMP2 and MMP9 (31). For example, a TATA box is present in the cis element of the MMP9 promoter, but is not present in the MMP2 promoter. The expression of MMP2 is mainly determined by the ubiquitous Sp-1 family of transcription factors. Thus, this diversity in the promoter compositions of MMP2 and MMP9 may be associated with the mechanism by which miR-212 differently regulated the expression of these proteins in ESCC cells.

Since increased miR-212 level was associated with poor outcome in patients with ESCC (12), the potential of therapeutic drugs to inhibit the role of miR-212 in promoting ESCC cell migration was investigated. Cell migration is controlled by several signaling cascades including PI3K/AKT, AMPK/ACC signaling, mTOR/p70S6K and estrogen receptor stress. Of all the drugs tested in the present study, berberine alone demonstrated an inhibitory effect on miR-212-induced KYSE-450 migration and EMT. Berberine was reported to attenuate cell migration and EMT in several types of cancer cells by targeting a number of signaling molecules and processes, including PI3K/AKT, p38, mTOR/p70S6K and AMPK-ERK (32-35). As an oncogene, miR-212 targets multiple molecules in the downstream signaling pathways to promote cell migration, invasion and EMT. These target genes include PTEN, SOX4 and Patched-1 (36-38).

In conclusion, the results from the present study demonstrate that miR-212 facilitates ESCC cell migration and invasion rather than proliferation, and berberine is a potential therapeutic drug against metastasis of ESCC in patients with high miR-212 expression. Further investigation of the mechanisms and its target gene by which miR-212 promotes ESCC cell migration and invasion is required.

\section{Acknowledgements}

Not applicable.

\section{Funding}

The present study was supported by Key Technologies R\&D Program of Science and Technology Commission Foundation of Henan Provence to BZ (grant no. 152102310110); Key Science and Technique Fund of Xinxiang to BZ (grant no. ZG15018); Xinxiang Medical University Student Innovation Fund to ZC (grant no. YJSCX201709Y); The First Affiliated Hospital of Xinxiang Medical University Foundation to YL (grant no. XYYFY2014BS-002); and The First Affiliated Hospital of Xinxiang Medical University Youth Foundation to BQ (grant no. QN-2017-A004).

\section{Availability of data and materials}

The datasets used and/or analyzed during the current study are available from the corresponding author on reasonable request.

\section{Authors' contributions}

ZC and YL contributed equally to this work. ZC and YL designed the methods and performed the majority of the experiments; BQ, CG, XW, LG and WY performed the migration experiments of the drug treatments and interpretation of the data; ZC, YL and BZ contributed to the conception and design of the study, and drafting the manuscript. All authors read and approved the manuscript.

\section{Ethics approval and consent to participate}

Not applicable.

\section{Patient consent for publication}

Not applicable.

\section{Competing interests}

The authors declare that they have no competing interests.

\section{References}

1. Ferlay J, Soerjomataram I, Dikshit R, Eser S, Mathers C, Rebelo M, Parkin DM, Forman D and Bray F: Cancer incidence and mortality worldwide: Sources, methods and major patterns in GLOBOCAN 2012. Int J Cancer 136: E359-E386, 2015.

2. Arnold M, Soerjomataram 1, Ferlay J and Forman D: Global incidence of oesophageal cancer by histological subtype in 2012 . Gut 64: 381-387, 2015.

3. Murphy G, McCormack V, Abedi-Ardekani B, Arnold M, Camargo MC, Dar NA, Dawsey SM, Etemadi A, Fitzgerald RC, Fleischer DE, et al: International cancer seminars: A focus on esophageal squamous cell carcinoma. Ann Oncol 28: 2086-2093, 2017.

4. Chen W, Zheng R, Zhang S, Zhao P, Li G, Wu L and He J: Report of incidence and mortality in China cancer registries, 2009. Chin J Cancer Res 25: 10-21, 2013.

5. Cheng J, Jin H, Hou X, Lv J, Gao X and Zheng G: Disturbed tryptophan metabolism correlating to progression and metastasis of esophageal squamous cell carcinoma. Biochem Biophys Res Commun 486: 781-787, 2017.

6. Croce CM: Causes and consequences of microRNA dysregulation in cancer. Nat Rev Genet 10: 704-714, 2009.

7. Bracken CP, Scott HS and Goodall GJ: A network-biology perspective of microRNA function and dysfunction in cancer. Nat Rev Genet 17: 719-732, 2016.

8. Sakai NS, Samia-Aly E, Barbera M and Fitzgerald RC: A review of the current understanding and clinical utility of miRNAs in esophageal cancer. Semin Cancer Biol 23: 512-521, 2013.

9. Harada K, Baba Y, Ishimoto T, Shigaki H, Kosumi K, Yoshida N, Watanabe $\mathrm{M}$ and $\mathrm{Baba} \mathrm{H}$ : The role of microRNA in esophageal squamous cell carcinoma. J Gastroenterol 51: 520-530, 2016.

10. Zhao BS, Liu SG, Wang TY, Ji YH, Qi B, Tao YP, Li HC and Wu XN: Screening of microRNA in patients with esophageal cancer at same tumor node metastasis stage with different prognoses. Asian Pac J Cancer Prev 14: 139-143, 2013.

11. Qi B, Liu SG, Qin XG, Yao WJ, Lu JG, Guo L, Wang TY, Li HC and Zhao BS: Overregulation of microRNA-212 in the poor prognosis of esophageal cancer patients. Genet Mol Res 13: 7800-7807, 2014.

12. Li B, Li J, Xu WW, Guan XY, Qin XY, Zhang LY, Law S, Tsao SW and Cheung AL: Suppression of esophageal tumor growth and chemoresistance by directly targeting the PI3K/Akt pathway. Oncotarget 5: 11576-11587, 2014. 
13. Lu Z, Peng K, Wang N, Liu HM and Hou G: Downregulation of p70S6K enhances cell sensitivity to rapamycin in esophageal squamous cell carcinoma. J Immunol Res 2016: 7828916, 2016.

14. Medina EA, Obertheu K, Polusani SR, Ortega V, Velagaleti GV and Oyajobi BO: PKA/AMPK signaling in relation to adiponectin's antiproliferative effect on multiple myeloma cells. Leukemia 28: 2080-2089, 2014.

15. Liang F, Wang YG and Wang C: Metformin inhibited growth, invasion and metastasis of esophageal squamous cell carcinoma in vitro and in vivo. Cell Physiol Biochem 51: 1276-1286, 2018.

16. Chen YZ, Bai N, Bi JH, Liu XW, Xu Go, Zhang LF, Li XQ and Huo R: Propranolol inhibits the proliferation, migration and tube formation of hemangioma cells through HIF-1 $\alpha$ dependent mechanisms. Braz J Med Biol Res 50: e6138, 2017.

17. Jiang SX, Qi B, Yao WJ, Gu CW, Wei XF, Zhao Y, Liu YZ and Zhao BS: Berberine displays antitumor activity in esophageal cancer cells in vitro. World J Gastroenterol 23: 2511-2518, 2017.

18. Livak KJ and Schmittgen TD: Analysis of relative gene expression data using real-time quantitative PCR and the 2(-Delta Delta C(T)) method. Method 5: 402-408, 2001.

19. Thiery JP, Acloque H, Huang RY and Nieto MA: Epithelialmesenchymal transitions in development and disease. Cell 139: 871-890, 2009.

20. Baker EA, Stephenson TJ, Reed MW and Brown NJ: Expression of proteinases and inhibitors in human breast cancer progression and survival. Mol Pathol 55: 300-304, 2002.

21. Rao JS, Gondi C, Chetty C, Chittivelu S, Joseph PA and Lakka SS Inhibition of invasion, angiogenesis, tumor growth and metastasis by adenovirus-mediated transfer of antisense uPAR and MMP-9 in non-small cell lung cancer cells. Mol Cancer Ther 4 1399-1408, 2005.

22. Wang YY, Tang LQ and Wei W. Berberine attenuates podocytes injury caused by exosomes derived from high glucose-induced mesangial cells through TGF $\beta 1-\mathrm{PI} 3 \mathrm{~K} / \mathrm{AKT}$ pathway. Eur J Pharmacol 824: 185-192, 2018.

23. Mishan MA, Ahmadiankia N, Matin MM, Heirani-Tabasi A, Shahriyari M, Bidkhori HR, Naderi-Meshkin H and Bahrami AR. Role of berberine on molecular markers involved in migration of esophageal cancer cells. Cell Mol Biol (Noisy-le-grand) 61: 37-43, 2015.

24. Chen CZ: MicroRNAs as oncogenes and tumor suppressors. N Engl J Med 353: 1768-1771, 2005.

25. Remenyi J, vanden Bosch MW, Palygin O, Mistry RB, McKenzieC, Macdonald A, Hutvagner G, Arthur JS, Frenguelli BG and Pankratov Y: miR-132/212 Knockout mice reveal roles for these miRNAs in regulating cortical synaptic transmission and plasticity. PLoS One 8: e62509, 2013

26. Remenyi J, Hunter CJ, Cole C, Ando H, Impey S, Monk CE, Martin KJ, Barton GJ, Hutvagner G and Arthur JS: Regulation of the miR-212/132 locus by MSK1 and CREB in response to neurotrophins. Biochem J 428: 281-291, 2010.
27. Jiping Z, Ming F,Lixiang W, Xiuming L, Yuqun S, Han Y,Zhifang L Yundong S, Shili L, Chunyan C and Jihui J: MicroRNA-212 inhibits proliferation of gastric cancer by directly repressing retinoblastoma binding protein 2. J Cell Biochem 114: 2666-2272, 2013.

28. Roomi MW, Kalinovsky T, Niedzwiecki A and Rath M: Modulation of uPA, MMPs and their inhibitors by a novel nutrient mixture in human colorectal, pancreatic and hepatic carcinoma cell lines. Int J Oncol 47: 370-376, 2015.

29. Yamashita K, Tanaka Y, Mimori K, Inoue H and Mori M: Differential expression of MMP and uPA systems and prognostic relevance of their expression in esophageal squamous cell carcinoma. Int J Cancer 110: 201-207, 2004.

30. Yan C and Boyd DD: Regulation of matrix metalloproteinase gene expression. J Cell physiol 211: 19-26, 2007.

31. Chakraborti S, Mandal M, Das S, Mandal A and Chakraborti T: Regulation of matrix metalloproteinases: An overview. Mol Cell Biochem 253: 269-285, 2003.

32. Kou Y, Li L, Li H, Tan Y, Li B, Wang K and Du B: Berberine suppressed epithelial mesenchymal transition through cross-talk regulation of PI3K/AKT and RAR $\alpha / R A R \beta$ in melanoma cells Biochem Biophys Res Commun 479: 290-296, 2016.

33. Li L, Wang X, Sharvan R, Gao J and Qu S: Berberine could inhibit thyroid carcinoma cells by inducing mitochondrial apoptosis, G0/G1 cell cycle arrest and suppressing migration via PI3K-AKT and MAPK signaling pathways. Biomed Pharmacother 95: 1225-1231, 2017.

34. Ai F, Chen M, Yu B, Yang Y, Xu G, Gui F, Liu Z, Bai X and Chen Z: Berberine regulates proliferation, collagen synthesis and cytokine secretion of cardiac fibroblasts via AMPK-mTOR-p70S6K signaling pathway. Int J Clin Exp Pathol 8: 12509-12516, 2015.

35. Kim HS, Kim MJ, Kim EJ, Yang Y, Lee MS and Lim JS: Berberine-induced AMPK activation inhibits the metastatic potential of melanoma cells via reduction of ERK activity and COX-2 protein expression. Biochem Pharmacol 83: 385-394, 2012.

36. Xie M, Fu Z, Cao J, Liu Y, Wu J,Li Q and Chen Y: MicroRNA-132 and microRNA-212 mediate doxorubicin resistance by down-regulating the PTEN-AKT/NF- $\mathrm{BB}$ signaling pathway in breast cancer. Biomed Pharmacother 102: 286-294, 2018.

37. Fu W, Tao T, Qi M, Wang L, Hu J, Li X, Xing N, Du R and Han B: MicroRNA-132/212 upregulation inhibits TGF- $\beta$-mediated epithelial-mesenchymal transition of prostate cancer cells by targeting SOX4. Prostate 76: 1560-1570, 2016.

38. Ma C, Nong $K$, Wu B, Dong B, Bai Y, Zhu H, Wang W, Huang X, Yuan $\mathrm{Z}$ and Ai K: miR-212 promotes pancreatic cancer cell growth and invasion by targeting the hedgehog signaling pathway receptor patched-1. J Exp Clin Cancer Res 33: 54, 2014.

This work is licensed under a Creative Commons Attribution-NonCommercial-NoDerivatives 4.0 International (CC BY-NC-ND 4.0) License. 